\title{
Positron Emission Tomography Measures of Benzodiazepine Receptors in Huntington's Disease
}

\author{
Vjera A. Holthoff, MD, *ł Robert A. Koeppe, PhD, ${ }^{*}$ Kirk A. Frey, MD, PhD, *† John B. Penney, MD, \\ Dorene S. Markel, MS, $\div$ David E. Kuhl, MD, ${ }^{*}$ and Anne B. Young, MD, PhD†
}

\begin{abstract}
We performed positron emission tomographic (PET) measurements of the regional distribution volume of benzodiazepine receptors and regional glucose metabolism in 6 drug-free patients with early Huntington's discase following injection of $\left[{ }^{11} \mathrm{C}\right]$ flumazenil, a nonsubtype selective central benzodiazepine receptor antagonist, and ${ }^{18} \mathrm{~F}$-2-fluoro-2deoxy-D-glucose, respectively. Flumazenil data were analyzed with a recently developed two-compartment, twoparameter tracer kinetic model. Benzodiazepine receptor density is related to distribution volume for flumazenil. In comparison with a group of healthy volunteers, benzodiazepine receptor density was significantly decreased in the caudate nucleus. Glucose metabolism was significantly reduced not only in the caudate nucleus but also in the putamen and thalamus. The changes in benzodiazepine receptor density observed in the caudate nucleus are commensurate with data obtained in postmortem autoradiographic studies of receptor density. Based on such postmortem studies we also anticipated changes in putamen and thalamic benzodiazepine receptor density. However, relatively little is known on receptor changes in early Huntington's disease, because the autoradiographic data available were obtained mostly in patients with advanced disease. The decreased glucose metabolism in the caudate and putamen agrees well with previously published results of PET studies, whereas metabolic impairment of the thalamus has not yet been described in Huntington's disease. The present study suggests that regional metabolism and $\gamma$-aminobutyric acid (GABA)benzodiazepine receptor changes in subcortical structures of patients with early Huntington's disease do not occur with the same time course: Caudate benzodiazepine receptor density is already severely impaired when other subcortical structures reveal only minor abnormalities. Impairment of neuronal metabolism seems to predate GABA/benzodiazepine receptor changes since the putamen and thalamus demonstrate metabolic impairment without detectable loss of benzodiazepine receptor density.
\end{abstract}

Holthoff VA, Koeppe RA, Frey KA, Penney JB, Markel DS, Kuhl DE, Young AB. Positron emission tomography measures of benzodiazepine receptors in Huntington's discase. Ann Neurol 1993;34:76-81

Huntington's disease (HD) is an autosomal dominant inherited disorder characterized by the onset in midlife of progressive motor and cognitive dysfunction. Previous studies with positron emission tomography (PET) and $\left[{ }^{18} \mathrm{~F}\right]$-2-fluoro-2-deoxy-D-glucose (FDG) in patients with symptomatic HD have shown marked declines in caudate and putamen glucose metabolism early in the disease and possibly even presymptomatically $[1-6]$. The decline in caudate and putamen FDG uptake correlated with the decline in functional capacity of the HD patients. Over $90 \%$ of caudate and putamen neurons use $\gamma$-aminobutyric acid (GABA) as a neurotransmitter [7] and these neurons send projections to the lateral and medial globus pallidus and the substantia nigra pars reticulata. Postmortem biochemical studies have revealed large decreases in GABA concentrations and glutamate decarboxylase (GAD; the enzyme that synthesizes GABA) activity in HD caudate and putamen as well as their projection zones $[8,9]$. In other postmortem studies of $\mathrm{ID}$, binding sites for both GABA and benzodiazepines have been found to be decreased in striatum and increased in substantia nigra [9-14]. Postmortem autoradiographic studies of GABA receptors labeled with competitive GABA agonists and benzodiazepine agonists (that bind to a modulatory site on the GABA receptor) have shown decreased binding in caudate and putamen and increased binding in globus pallidus $[15-18]$. This latter increase presumably reflects a compensatory upregulation of pallidal receptors in response to denervation. In the superficial layer of the midfrontal cortex, total benzodiazepine receptor (BZR) binding was increased but its enhancement by GABA and barbiturates was significantly reduced [17]. We have now utilized the
From the *Department of Internal Medicine, Division of Nuclear Medicine and the $\doteqdot$ Department of Neurology, University of Michi-

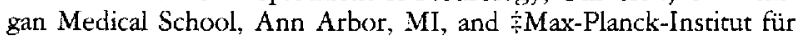
Neurologische Forschung, Köln, Federal Republic of Germany.
Received Jul 11, 1991, and in revised form Sep 11, 1992, and Jan 29, 1993. Accepted for publication Feb 8, 1993.

Address correspondence to Dr Holthoff, Max-Planck-Institut für Neurologische Forschung, Gleueletstr. 50, 5000 Köln 41, FRG. 
benzodiazepine antagonist $\left[{ }^{11} \mathrm{C}\right]$ flumazenil (FMZ) to examine BZRs by PET in living HD patients, and compared the relative sensitivity of the method to measurements of glucose metabolism in the same subjects.

\section{Materials and Methods \\ Subjects}

Six patients (mean age, $53 \pm 9$ years; 3 men, 3 women) with adult-onset HD underwent neurological examination and evaluation of total functional capacity (TFC) according to the Shoulson and Fahn scale [19] prior to the PET studies. The diagnosis of HD was made on the basis of the family history, physical examination, laboratory tests to exclude other diseases, and the findings on computer tomographic $\left(\mathrm{C}^{\prime} \mathrm{T}\right)$ or magnetic resonance imaging (MRI) scans. All patients studied here exhibited little or no cerebral atrophy as measured by cranial CT or MRI. TFC assesses the patient's ability to be gainfully employed, to handle financial affairs, to manage domestic responsibilities, to perform activities of daily life, and to be cared for at home. The TFC scale ranges from 13 to 0 , with 13 being normal and 0 indicating total incapacitation. Patients were in stage $I(n=3)$ or stage II $(\mathrm{n}=3)$, with a TFC for the group of $10.7 \pm 1.6$ (mean \pm standard deviation). The estimated duration of symptoms was approximately 3 years in the stage I patients and 6 years in the stage II patients. They were compared with 6 normal control subjects (mean age, $50 \pm 13$ years) for the receptor studies and with 17 normal control subjects (mean age, $52 \pm 10$ years) for glucose metabolism studies. Control subjects were free of significant general medical, neurological, and psychiatric illness and had no family history of first-degree relatives with familial neurologic or psychiatric disease. They were also screened to exclude history of head trauma with loss of consciousness, drug or alcohol abuse, or dependence and excessive consumption of caffeine. They were free of prescribed sedatives, tranquilizers, and anxiolytics for 12 months prior to participation. The studies were approved by the University of Michigan Institutional Review Boards and written informed consent was obtained for all studies.

\section{Positron Emission Tomograpby Imaging}

A radial artery and cutaneous vein were cannulated for withdrawal of blood samples and for injection of the radioisotopes, respecrively. The subjects were positioned with their eyes and ears unoccluded in the gantry of a CTI/Siemens 931/08-12 tomograph (Siemens Gammasonics, Inc, Hoffman Estates, IL). The scanner simultaneously acquires 15 contiguous transaxial slices covering approximately a $10-\mathrm{cm}$ axial field of view, with an inplane spatial resolution of approximately $7-$ to $8-\mathrm{mm}$ full width at half maximum.

The 6 patients were first studied following injection of $22.5 \mathrm{mCi}\left[{ }^{11} \mathrm{C}\right] \mathrm{FMZ}$ and then underwent a second PET study following injection of $10 \mathrm{mCi} F D G$. The radioisotope injections were separated by 100 minutes to allow decay and washout of residual isotope. Six age-matched control subjects underwent a single study following injection of $22.5 \mathrm{mCi}$ $\left.{ }^{11} \mathrm{C}\right] \mathrm{FMZ}$. Another 17 age-matched control subjects were examined with FDG only.

A dynamic series of PET data ( 15 frames) and 30 to 35 arterial blood samples were obtained over 60 minutes for the FMZ studies. Local cerebral metabolic rates for glucose (LCMRG) were calculated using a three-compartment model and a single-scan approximation with gray matter kinetic constants derived from normal subjects and a lumped constant of 0.54 . A calculated attenuation correction was applied to all image reconstructions.

\section{Correction of the Arterial Input Function for Flumazenil}

The time-course of $\left[{ }^{11} \mathrm{C}\right] \mathrm{FMZ}$ following injection was determined in arterial plasma with the use of an Nal (En ${ }^{3}$ hance, New England Nuclear, Boston, MA) well counter. Thirteen samples for each study were processed by liquid chromatography using Sep-Pak $C_{18}$ (Waters, Milford, MA) cartridges to separate authentic FMZ from labeled metabolites as described previously [20]. The arterial blood was centrifuged and the plasma was applied to the column. A metabolite and an FMZ fraction were then obtained for each sample by prewashing the column with $9 \mathrm{ml}$ of a $35 \%$ methanol/sodium phosphate buffer solution and $5 \mathrm{ml}$ of $100 \%$ methanol, respectively. The separation was corrected for loss of authentic FMZ with the metabolite fraction on the basis of $\left[{ }^{3} \mathrm{H}\right] \mathrm{FMZ}$ added as internal standard to each sample prior to processing. The ratio of $\left[{ }^{11} \mathrm{C}\right] \mathrm{FMZ}$ to $\left[{ }^{11} \mathrm{C}\right]$ metabolites was determined in the well counter and the recovery of $\left.{ }^{3} \mathrm{H}\right] \mathrm{FMZ}$ was determined by liquid scintillation spectrometry. The arterial input function was then corrected for the metabolite fraction.

\section{Realignment of the Images}

Reconstructed images were realigned with the use of small molecular sieve beads as fiduciaries to correct for subject motion during and between scanning sessions. One bead was placed on the forehead and one behind each ear, and they stayed tightly attached to the scalp during the entire scanning period. Approximately $1 \mu \mathrm{l}$ of the ligand preparation was pipetted onto each bead as follows: $6 \mu \mathrm{Ci}\left[{ }^{11} \mathrm{C}\right]$ per bead prior to a receptor study and $1.5 \mu \mathrm{Ci}\left[{ }^{18} \mathrm{~F}\right]$ prior to an $\mathrm{FDG}$ study. Following reconstruction of the dynamic PET sequence, each bead was marked with a cursor on the 7.5- to 10.0-minute frame and the $(x, y, z)$ coordinates were calculated based on the radioactivity distribution in the vicinity of the beads. This frame defined the standard orientation. An automated routine then located and calculated the $(x, y, z)$ coordinates for each bead in the other 14 frames. These frames were then realigned to the orientation of the standard frame. With this method, it is possible to correct for the three translational and three rotational degrees of freedom. Inplane ( $\mathrm{x}$ and $\mathrm{y}$ ) translation error is smaller than 0.1 pixel (approximately $0.15 \mathrm{~mm}$ ), the axial translation error (z) is smaller than 0.1 plane (approximately $0.5 \mathrm{~mm}$ ), and the rotation errors $(x y, x z, y z)$ are smaller than 0.2 degree. Good realignment was achieved in all studies.

\section{Compartmental Analysis of $\left(^{11} \mathrm{C}\right)$ Flumazenil \\ Transport and Distribution}

The dynamic series of emission scans obtained from the receptor studies was analyzed together with the metabolitecorrected arterial input curve on the basis of a twocompartment, two-parameter model. The model consists of an intravascular compartment and a single tissue compart- 
ment accounting for the combined tracer activity of free, nonspecific, and specific binding pools. The estimated parameters are the ligand transport from blood to brain, $\mathrm{K}_{1}$ (milliliter of plasma/milliliter of brain/min) and the clearance rate from tissue back to blood, $\mathrm{k}_{2}\left(\mathrm{~min}^{-1}\right)$. The ratio of these two parameters provides an estimate of the ligand's distribution volume (DV) in the brain, which is assumed to represent predominantly specific BZR binding. As shown previously [21], DV is linearly related to $B L R$ density $\left(B_{\text {max }}, \mathrm{pmol}^{-1}\right.$ min ${ }^{-1}$. Parametric images of $K_{1}$ and $D V$ were generated for each study on a pixel-by-pixel basis using a weighted integral technique similar to that used for the cerebral blood flow studies.

\section{Data Analysis}

Regions of interest were determined on the $K_{1}$ images obtained from the FMZ study and were directly superimposed on the corresponding images of the DV maps and FDG scans. The regions included the frontal cortex, the occipital cortex, thalamus, caudate, putamen, cerebcllar cortex, and the pons. Values from structures identified in both hemispheres were averaged for each subject.

Based on previously published autoradiographic studies $[15-18]$ as well as PET measurements of glucose metabolism [1-6], we were primarily interested in changes in the caudate nucleus, as well as the putamen and the thalamus. An a priori hypothesis predicted decreases in caudate and putaminal DV and glucose metabolic rate. $\mathrm{K}_{1}$, which is largely proportional to regional cerebral blood flow, was expected to show similar changes as seen for regional glucose metabolism. Due to either conflicting results on changes in thalamic glucose metabolism in $\mathrm{HD}[2,3,5,6]$ or lack of previous reports on alterations in thalamic $\mathrm{B} Z \mathrm{R}$ distribution, it was difficult to anticipate any changes for that region. Paired Student's $t$ tests were applied for comparison of regional mean values for transport rate and distribution volume for FMZ in the patient and control groups. The change in the metabolic rate of the caudate and putamen in the HD patients (expressed as the percentage of the mean of the control patients) was compared to the change in the FMZ DV in the same regions (also expressed as a percentage of the control means) by analysis of variance (ANOVA).

\section{Results}

The Table summarizes the regional values for radioligand transport $(\mathrm{K}$ ) and $\mathrm{BZZR}$ DV obtained for the $\left[{ }^{11} \mathrm{C}\right] \mathrm{FMZ}$ studies in both patient and control groups. $\mathrm{K}_{1}$ in both groups varied slightly from region to region and was highest in the thalamus, intermediate in the visual cortex and putamen, and lowest in the frontal cortex and posterior fossa. In $\mathrm{HD}, \mathrm{K}_{1}$ in the caudate was reduced by $17 \%(p=0.02)$ when comparing patient and control groups. Furthermore, $\mathrm{K}_{1}$ values revealed a nonsignificant decrease in the putamen by $9 \%$, whereas in all other regions analyzed they were slightly increased.

DV (milliliter of plasma/milliliter of tissue) varied about fivefold between regions (see Table). As previously observed [20], the highest receptor densities in patients and control subjects were found in the frontal and visual cortices, followed by the putamen, thalamus, and cerebellar cortex. The lowest values were demonstrated in the pons. Values of DV were lower in patients with HD than in control subjects in all supratentorial regions but the decrease reached significance only in the caudate ( $26 \%$ reduction, $p=0.02$ ).

The FDG scans were carried out in the same HD patients as were the FMZ scans but they were compared to a different group of 17 age-matched normal control subjects. Glucose metabolism in the HD group was significantly decreased in the caudate and putamen by $47 \%$ and $41 \%$, respectively ( $p<0.0001$ ), and also in the thalamus by $18 \%(p=0.00 \mathrm{l})$. The comparison of regional ratios for LCMRG revealed a reduction in caudate metabolic rate $(47 \%)$ that was more substantial than the decrease in FMZ DV $(26 \% ; \mathrm{F}(1,10)=$ $17.272 ; p=0.0(1)$.

\section{Discussion}

Assessment of $B Z R$ density in living humans is now possible through the use of radiolabeled ligands and dynamic PET [20-25]. Central-type BZRs are closely related to GABA receptors, and both receptors exist on a family of ligand-gated chloride channels. GABA is the most important inhibitory neurotransmitter in the brain and its inhibitory function is mediated by changes in the chloride conductance of the neuronal membrane. Benzodiazepines increase the frequency of chloride channel openings, while the duration of opening events and channel conductance are not altered (for a review, see $[26,27])$.

$\left[{ }^{11} \mathrm{C}\right]$ Flumazenil is a nonsubtype selective, centraltype $\mathrm{BZ}$. R antagonist with a high relative proportion of specific, receptor-mediated retention in the brain [22-24]. It is devoid of significant intrinsic pharmacological activity, but potently antagonizes the effects of benzodiazepines. As demonstrated recently, a twocompartment, two-parameter tracer kinetic model provides precise estimations of the kinetic behavior of $\left[{ }^{11} \mathrm{C}\right] \mathrm{FM} Z$ in the human brain $[20,21,25]$ and makes it possible to obtain pixel-by-pixel maps of radioligand transport $\left(\mathrm{K}_{1}\right)$ and receptor density (DV). DV is considered to represent predominantly specific $\mathrm{B}^{\prime} Z \mathrm{R}$ binding. Allosteric interactions between GABA and BZRs have been described (agonist binding to one receptor increases agonist binding to the other [26]), but as an antagonist, FMZ in vitro binding has been shown to be unaffected by endogenous or added GABA or barbiturates [28, 29],

The pathology in HD is profound in the caudate and putamen but not all striatal neurons die as a consequence of the inherited gene defect. As many as $90 \%$ of striatal neurons are projection neurons, contain GABA, and are medium spiny neurans $[7,14]$. GABAergic striatal output neurons appear to inhibit 


\begin{tabular}{|c|c|c|c|c|}
\hline \multirow[b]{2}{*}{ Regions } & \multicolumn{2}{|c|}{ Patients } & \multicolumn{2}{|c|}{ Control Subjects } \\
\hline & $\mathrm{K}_{1}$ & DV & $\mathrm{K}_{1}$ & DV \\
\hline Caudate & $0.28 \pm 0.03^{b}$ & $1.62 \pm 0.14^{\mathrm{b}}$ & $0.33 \pm 0.04$ & $2.19 \pm 0.48$ \\
\hline Putamen & $0.33 \pm 0.03$ & $2.87 \pm 0.27$ & $0.36 \pm 0.03$ & $3.04 \pm 0.51$ \\
\hline Thalamus & $0.41 \pm 0.03$ & $2.71 \pm 0.25$ & $0.39 \pm 0.05$ & $2.96 \pm 0.41$ \\
\hline Frontal cortex & $0.31 \pm 0.03$ & $4.20 \pm 0.62$ & $0.30 \pm 0.02$ & $4.57 \pm 0.75$ \\
\hline Visual cortex & $0.36 \pm 0.06$ & $4.99 \pm 0.71$ & $0.35 \pm 0.05$ & $5.74 \pm 1.04$ \\
\hline Cerebellum & $0.33 \pm 0.06$ & $3.24 \pm 0.37$ & $0.32 \pm 0.06$ & $3.25 \pm 0.30$ \\
\hline Pons & $0.35 \pm 0.04$ & $1.09 \pm 0.14$ & $0.31 \pm 0.05$ & $1.01 \pm 0.10$ \\
\hline
\end{tabular}

${ }^{a}$ Data are means \pm standard deviations, in milliliters of plasma/milliliter of tissue $/ \mathrm{min}$.

${ }^{\mathrm{b}} p=0.02$ by two-tailed Student's $t$ test.

GABAergic neurons in the globus pallidus and substantia nigra, which in turn project to the thalamus and brainstem (for review, see [30]). Somatostatin and acetylcholine interneurons are relatively spared and the brunt of the pathology falls on the GABAergic projection neurons. As shown in postmortem autoradiographic studies in patients with $\mathrm{HD}$, the consequences of the loss of these projection neurons are caudate and putamen atrophy and compensatory upregulation of GABA receptors and BZRs [16] in the neurons of the denervated projection zones. In advanced cases of $H D$ $(>10$ years' duration) GABA and BZR density are reduced by approximately 44 and $55 \%$, respectively, in the caudate and putamen. GABA receptors are increased by about $106 \%$ and BZRs, by $72 \%$ in the lateral globus pallidus [18]. Although the exact cellular location of BZR measured autoradiographically is not known, this increase is believed to be an attempt of the deafferentated neurons to maintain homeostasis by increasing their functional response to a decreased synaptic input [16]. In early HD (stages I and II) only two postmortem cases have been examined and in those BZR density was reduced significantly by $44 \%$ in the putamen and increased by $58 \%$ in the lateral globus pallidus [16].

This is the first study of BZRs in living individuals with HD. PET measurements of BZR density and glucose metabolism were made in 6 patients with early $\mathrm{HD}$ (stages I and II) following injection of $\left[{ }^{11} \mathrm{C}\right] \mathrm{FMZ}$ and $\left[{ }^{18}\right.$ F]FDG, respectively. We examined BZR DV in cortical as well as subcortical regions and our particular interest was directed toward possible changes in the metabolic rate and BZR density in the caudate, putamen, and thalamus when comparing patients to healthy control subjects. The PET technique is excellent for measuring metabolic and neurochemical parameters early in a disease process without confounding factors of drug effects or other illnesses. It would also have been of interest to examine pallidal BZRs in vivo and to verify the changes described in postmortem studies of advanced HD and also two brains with early HD [15-18]. One of the restrictions of the PET technique for in vivo studies of basal ganglia, however, is that accurate measurements of globus pallidus activity are not possible due to its small volume, scanner resolution, and difficulty discriminating between putamen and pallidum in our PET images. The globus pallidus is threefold smaller than the putamen, and its volume is approximately $40 \%$ below that required for a structure to be measured accurately with our scanner resolution [31].

We found that radioligand transport $\left(\mathrm{K}_{1}\right)$ and BZR DV in the patient group are significantly decreased only in the caudate nucleus and did not reach significance in the putamen and thalamus. Glucose metabolism, however, is significantly reduced in the caudate as well as in the putamen and thalamus. The present study reveals findings and results that corroborate some of the previously published observations in HD. The changes observed in BZR density and glucose metabolism in the caudate nucleus are commensurate with previously reported results of postmortem autoradiographic studies [15-18] and with PET measurements of glucose metabolism [1-6], respectively. It has been described that in early stages of HD, as in the patients presented here, caudate metabolism is affected more dramatically than is putamen metabolism [3] and pathological findings reveal that the disorder begins in the caudate nucleus and then progresses to the putamen [15]. Although the patients studied here exhibited little or no cerebral atrophy as measured by cranial CT or MRI, it should be considered that the changes found for the caudate nucleus may arise either by loss of binding sites and decreased neuronal function or by atrophy and partial volume artifact. The latter, however, is not supported by the fact that the change in $\mathrm{K}_{1}$ is far smaller than the changes observed for DV and giucose metabolic rate. Changes reflecting merely cell loss would be expected to effect both transport rate and glucose metabolism to a similar degree. The 
modest decrease in putaminal DV differs from postmortem autoradiographic results that have described a pronounced decrease in BZR density. However, postmortem data were found in patients with advanced $\mathrm{HD}$, and only 2 with early HD have been studied so far [16]. It could be that in carly HD the typical decrease in putaminal BZR density has not yet occurred. Walker and colleagues [16] reported that putaminal GABA and BZR levels in 2 parients with early HD (without obvious neuronal cell loss) were intermediate between those of control subjects and parients with advanced HD. The pallidal BZR DV was increased in these early cases almost to the level seen in advanced HD [16]. We also considered the possibility that our measured putamen values were artificially high because of partial volume averaging of the pallidum (which is expected to show increased receptor density) and insula that may be contributing activity to the putamen. We have performed simulation studies, however, that do not support this hypothesis. Computer-generated PET data sets were produced simulating the isotope distribution found in $\left[{ }^{11} \mathrm{C}\right] \mathrm{FMZ}$ receptor scans using a computerized digital brain phantom as described previously [32].

Thalamic glucose metabolism is significantly reduced, but measurements of $\mathrm{K}_{1}$ and $\mathrm{DV}$ in the $\left[{ }^{11} \mathrm{C}\right] \mathrm{FMZ}$ scans show no significant change. Previous studies either have failed to show a significant change in thalamic metabolism $[2,5,6]$ or have demonstrated increased metabolism [3]. Metabolic impairment of the thalamus in HD could be the result of selective neuronal degeneration. Dom and colleagues [33] demonstrated a $50 \%$ decrease in the small-cell population of the ventrolateral group of the thalamus with a preservation of the mactoneuronal population. The small cells constitute 25 to $35 \%$ of the neuronal population in this region. Decreased thalamic BZR density was revealed previously in a postmortem autoradiographic study of advanced HD only [15] and in rats following striatal kainic acid lesions [34]. More advanced or severe striatal pathology may be necessary for the production of secondary thalamic receptor changes.

The present study suggests that regional metabolic and GABA/BZR changes in subcortical structures of HD patients do not occur with the same time course. Impairment of neuronal metabolism seems to predate GABA/BZR changes in stage I and II patients. Caudate glucose metabolism and BZR density are already severely impaired when other subcortical structures reveal only minor abnormalities. It will be important to determine the relationship of BZR changes to cognitive and motoric symptoms in this progressive disease. Possibly, the decline in $\mathrm{BZR}$ density might provide a more accurate estimate of disease progression than reductions in glucose metabolism.

\section{References}

1. Kuhl DE, Phelps ME, Markham $\mathrm{CH}$, et al. Cerebral metabolism and atrophy in Huntington's disease determined by ${ }^{18} \mathrm{FDG}$ and computed tomographic scan. Ann Neurol 1982;12:425-434

2. Kuhl DE, Markham CH, Metter EJ, et al. Local cerebral giucose utilization in symptomatic and presymptomatic Huntington's disease. In: Sokoloff L, ed. Brain imaging and brain funcrion. New York: Raven, 1985:199-209

3. Young AB, Penney JB, Starosta-Rubinstein S, et al. PET scan investigation of Huntington's disease: cerebral metabolic correlates of neurologic features and functional decline. Ann Neurol 1986;20:296-303

4. Mazziotta JC, Phelps M, Pahl J, et al. Reduced cerebral glucose metabolism in asymptomatic subjects at risk for Huntington's disease. N Engl J Med 1987;316:357-362

5. Grafton ST, Mazziotta JC, Pahl JJ, et al. A comparison of neurological, structural, and genetic evaluations in persons at risk for Hunrington's disease. Ann Neurol 1990;28:614-621

6. Kuwert T, Lange HW, Langen K-J, et al. Cortical and subcortical glucose consumption measured by PET in patients with Huntington's disease. Brain 1990;113:1405-1423

7. Ribac CE, Vaughn JE, Roberts E. The GABA neurons and their axon terminals in rat corpus striatum as demonstrated by $\mathrm{GAD}$ immunocytochemistry. J Comp Neurol 1979;187:261-284

8. Perry TL, Hansen S, Kloster M. Huncington's chorea: deficiency of gamma-aminobutyric acid in brain. N Engl J Med 1973; 228:337-342

9. Iverson LL, Bird E, Spokes EGS, et al. Agonist specificity of GABA binding sites in human brain and GABA in Huntington's disease and schizophrenia. In: Krogsgaard-Larson P, ScheelKruger J, Kofod H, eds. GABA neurotransmitter. New York: Academic, 1978:179-190

10. Enna SJ, Bennett JP Jr, Bylund DB, et al. Alterations of brain neurotransmitter receptor binding in Huntington's chorea. Brain Res 1976;116:531-537

11. Lloyd KG, Dreksler S, Bird ED. Alterations in $\left[{ }^{3} \mathrm{H}\right]-\mathrm{GABA}$ binding in Huntington's chorea. Life Sci 1976;21:747-754

12. Reisine TD, Beaumont K, Bird ED, et al. Huntington's disease: alterations in neurotransmitter receptor binding in the human brain. Adv Neurol 1979;23:717-726

13. Reisine TD, Wastek GJ, Speth RC, et al. Alterations in the benzodiazepine receptor of Huntington's diseased human brain. Brain Res 1979;165:183-187

14. Waddington JL, Cross AJ. Characterization of denervation supersensitivity in the striatonigral GABA pathway of the kainic acid-lesioned rat and Huntington's disease. Brain Res Bull $1980 ; 5: 825-828$

15. Penney JB, Young AB. Quantitative autoradiography of neurotransmitter receptors in Huntington's disease. Neurology 1982;32:1391-1395

16. Walker FO, Young AB, Penney JB, Dovorini-Zis K. Benzodiazepine and GABA receptors in early Huntington's disease. Neurology 1984;34:1237-1240

17. Whitehouse PJ, Trifiletti RR, Jones BE, et al. Neurotransmitter receptor alterations in Huntington's disease: autoradiographic and homogenate studies with special reference to benzodiazepine receptor complexes. Ann Neurol 1985;18:202-210

18. Penney JB, Pan HS. Quantitative autoradiography of GABA and benzodiazepine binding in studies of mammalian and human basal ganglia function. In: Boast CA, ed. Quantitative receptor autoradiography. New York: Liss, 1986:29-52

19. Shoulson I, Fahn S. Huntington's disease: clinical care and evaluation. Neurology 1979;29:1-3

20. Frey KA, Holthoff VA, Koeppe RA, et ai. In vivo quantification of cerebral benzodiazepine binding with $\left[{ }^{11} \mathrm{C}\right]$ flumazenil and positron emission tomography: distinct images of ligand trans- 
port and binding on the basis of simplified compartmental analysis. Ann Neurol 1991;30:663-672

21. Koeppe RA, Holthoff VA, Frey KA, et al. Compartmental analysis of $\left[{ }^{11} \mathrm{C}\right]$ flumazenil kinetics for the estimation of ligand transport rate and receptor distribution. J Cereb Blood Flow Metab 1991;11:735-744

22. Persson A, Ehrin E, Eriksson $L$, et al. Imaging of $\left[{ }^{11} \mathrm{C}\right\}$-labelled Ro 15-1788 binding to benzodiazepine receptors in the human brain by positron emission tomography. J Psychiarr Res 1985; 19:609-622

23. Shinotoh H, Yamasaki $T$, Inoue $O$, et al. Visualization of specific binding sites of benzodiazepine in human brain. $J$ Nucl Med $1986 ; 27: 1593-1599$

24. Pappata S, Samson Y, Chavoix C, et al. Regional specific binding of $\left[{ }^{11} \mathrm{C}\right\} \mathrm{Ro}$ 15-1788 to central rype benzodiazepine receptors in human brain: quantitative evaluation by PET. J Cereb Blood Flow Metab 1988;8:304-313

25. Holthoff VA, Koeppe RA, Frey KA, et al. Differentiation of radioligand delivery and binding in the brain: validation of a two-compartment model for [ $\left.^{11} \mathrm{C}\right]$ Humazenil. J Cereb Blood Flow Metab 1991;11:745-752

26. Moehler H, Richards J. Receptors for anxiolytic drugs. In: Mallic JB, Enna SJ, Yamamura HI, eds. Anxiolytics. Neurochemical, behavioural and clinical perspectives. New York: Raven, $1983: 15-40$

27. Tuyman RE, Rogers CJ, MacDonald RL. Differential regulation of $y$-amino-butyric acid receptor channels by diazepine and phenobarbital. Ann Neurol 1989;25:213-220

28. Farb DH, Borden LA, Chan CY, et al. Modulation of neuronal function through benzodiazepine receptors: biochemical and electrophysiological studies of neurons in primary monolayer cell culture. Proc Nacl Acad Sci USA 1984;435:1-31

29. Möhler H, Richards JG. Agonist and antagonist benzodiazepine receptor interaction in vitro. Nature 1981;294:763-765

30. Albin RL, Young AB, Penney JB. The functional anatomy of the basal ganglia. Trends Neurosci 1989;12:366-375

31. Mazziotta JC, Phelps ME, Plummer D, Kuhl DE. Quantiration in positron emission computed tomography: S. Physicalanatomical effects. J Compur Assist Tomogr 1981;5:734743

32. Hutchins GD. Physiologic signal detection in positron emission tomography. In: Kuhl DE, ed. Frontiers in nuclear medicine: in vivo imaging of neurotransmitter functions in brain, heart and tumors. Washington, DC: American College of Nuclear Physicians, 1991:141-155

33. Dom R, Malfroid M, Baro F. Neuropathology of Huntington's chorea: cytometric studies of the ventrobasal complex of the thalamus. Neurology 1976;26:64-68

34. Pan HS, Young AB, Penney JB. Changes in $\left[{ }^{3} \mathrm{H}\right\}$ muscimol binding in substantia nigra, entopeduncular nucleus, and thalamus after striatal lesions as demonstrated by quantitative receptor autoradiography. Soc Neurosci Abstr 1981;7:500 